Th. W. Engelmann: Ueber den Bau der quergestreiften Substanz etc. $\mathbf{5} 31$

in die Blutbahn gebrachten Peptons, je nachdem es vom Darme aus resorbirt oder direkt in das Blut gebracht wird, bezweifeln. Ich will nur darauf hinweisen, dass es mir nicht zur Pflicht gemacht werden kann, die Untersuchungen von Schmidt-Mülheim, Fano und von Hofmeister nach der physiologischen Bedeutung des Peptons mit den meinigen in directen Verband $\mathrm{zu}$ bringen.

\title{
Ueber den Bau der quergestreiften Substanz an den Enden der Muskelfasern.
}

Von

\author{
Th. W. Engelmann \\ in Utrecht.
}

Mit 1 Holzschnitt.

Aus verschiedenen morphologischen und physiologischen Gesichtspunkten ist eine Beantwortung der Frage erwünscht, wie die quergestreifte Substanz am Ende der Faser gebaut sei. Obschon, soviel mir bekannt, ein Versuch, diese Frage zu entscheiden, bisher nicht gemacht ist, weiss doch wohl ein Jeder, der sich näher mit dem mikroskopischen Bau der Muskelfasern beschäftigt hat, dass im Allgemeinen irgendwie auffällige Eigenthümlichkeiten dieses Banes am Faserende nicht bestehen: die Querstreifung setzt sich bis ans Ende des Sarkolemmrobres anscheinend unverändert fort. Die Frage kann zunächst nur sein : endigt die Muskelsubstanz stets mit der gleichen Substanzschicht oder nicht, und dann: zeigt die terminale Schicht vielleicht doch feinere, für sie als solche charakteristische Besonderheiten?

Die weitgehende Uebereinstimmung'im feineren Bau der quergestreiften Substanz bei Muskeln von den verschiedensten Lokalitäten und den verschiedensten Thieren, die strenge Gesetzmässig- 
keit, mit der die vielen verschiedenartigen Schichten tiberall im Verlauf der Faser in gleichem Wechsel aufeinander folgen, liessen erwarten, dass die Endigungsweise überall wesentlich dieselbe sein werde. So lange man nur zweierlei Schichten, eine anisotrope (Hauptsubstanz) und eine isotrope (Zwischensubstanz) unterschied, würde es sich hiernach nur darum gehandelt haben, welche dieser beiden als terminale Schicht auftrete. Jetzt, wo wir wissen, dass die erstere stets noch aus zwei Querscheiben und einer Mittelscheibe, die letztere aus einer Zwischenscheibe, zwei Nebenscheiben and vier Lagen plasmatischer ${ }^{1}$ ) Substanz zusammengesetzt ist, muss die Untersuchung bis auf diese Unterabtheilungen herabgehen. Selbstverständlich ist man hierdurch in noch höherem Maasse in der Wahl der Untersuchungsobjecte beschränkt, als sonst ohnehin schon der Fall gewesen sein würde. Nur sehr hochfächrige, nicht dicke Elemente, also Fasern von Arthropoden, namentlich von Käfern, versprachen brauchbare Resultate. Und auch unter diesen wesentlich nur solche Fasern, deren Enden nicht in dünne kegelförmige Spitzen oder verschwindend dünne keilförmige Platten ausliefen, sondern durch eine möglichst senkrecht zur Längsachse der Faser stehende Fläche von mässiger Ausdehnung begrenzt waren. Denn nur in letzterem Falle durfte auf erfolgreiche Anwendung des hier sehr wichtigen Polarisationsapparates gerechnet werden.

Als braucbbare Objecte kann ich, wie schon früher für das Studium der Contractionserscheinungen, die sehr dünnen, meist platten (oft allerdings an den Enden auch $\mathrm{zu}$ platten) Fasern empfehlen, welche die Abdominalsegmente von Telephorus melanurus mit einander verbinden. Näheres über dieselben und ihre Präparationsweise wurde früher schon angegeben. Uebrigens

1) Mit diesem, unlängst von Merkel (Arch. f. mikr. Anat., Bd. XIX, p. 649), übrigens in nicht durchaus gleichem Sinne, verwendeten Ausdruck will ich in Zukunft die helle isotrope Substanz bezeichnen, welche in der ruhenden Faser die Zwischenscheibe von der Nebenscheibe, und diese von der Querscheibe trennt, wobei noch dahin gestellt bleiben mag, ob sie an beiden Stellen identisch ist. Ich betone übrigens, dass mit dem Ausdruck "plasmatisch" nicht gesagt sein soll, dass diese Substanz eine Flüssigkeit sei. Dies wird zwar von manchen Forschern angenommen, aber ich kann nicht finden, dass die Thatsachen dazu nöthigen. Soviel ist nur sicher, dass jene Schicht ganz ausserordentlich wasserreich ist. 
lieferten auch Muskelfasern von anderen Körperstellen (Thorax und Extremitäten, Genitalapparat) und von anderen Käfern (u. a. Chrysomela, Trichius, Phyllopertha, Gnorimus, Hoplia, Carabus, Passalus) mancherlei völlig brauchbare Präparate, stellenweise sogar bessere als Telephorus. Selbstverständlich müssen die Muskelfasern in unversehrter Verbindung mit ihren Sehnen, bezïglich, wo diese fehlen, mit den Chitinhäuten oder -stücken, an denen ihre Enden fixirt sind, isolirt werden. Vorausgehende gute Erhärtung, am besten des ganzen Thieres, in starkem Alkobol, Osmiumsäure, Salicylsäure u. dgl. ist nöthig; sehr empfehlenswerth, die bei vorläufiger Durchmusterung mit schwächerer Vergrösserung Gutes versprechenden Gewebsstiuckchen, unmittelbar nach dem Einlegen in Balsam oder verdünntes Glycerin, unter dem einfachen Mikroslsop mit spitzen Nadeln so weit nöthig für genaue Prüfung mit stärkster Vergrösserung zugänglich zu machen.

Es ist wünschenswerth, zunächst solche Faserenden zu suchen, welche das Bild des Ruhezustandes zeigen. Denn allein in diesem sind alle verschiedenen Schichten deutlich unterscheidbar. Ich habe allmählich gegen hundert völlig brauchbare derartige Stellen zu Gesicht bekommen und genau geprift. Eins dieser Präparate (aus dem Thorax von Chrysomela fastuosa) zeigt nebenstehender Holzschnitt bei etwa $800-$ maliger Vergrösserung ${ }^{1}$ ).

Bei allen Fasern endigte die quergestreifte Substanz in wesentlich gleicher Weise, mit isotroper, speciell plasmatischer Substanz. Und zwar war in dieser Schicht ausnahmslos eine Nebenscheibe vorhanden, die weder nach Dimensionen, Form und Bau, noch nach optischen Eigenschaften (sie war durchaus isotrop) und Tinktionsfähigkeit von den anderen Nebenscheiben derselben Faser sich unterschied.

Die plasmatische Substanz zwischen der Nebenscheibe und dem Faserende, also die eigentliche terminale Schicht, war in vielen Fällen (vgl. z. B. den Holzschnitt) nicht dicker, wie die entsprechende Schicht zwischen Neben- und

1) Zur Erläuterung des Holzschnittes noch Folgendes. Die Faser war in Alkohol erhärtet und nach schwacher Pikrocarminfärbung in Balsam eingeschlossen. Im Polarisationsapparat erschienen nur die Quer- und Mittel- 
Zwischenscheibe weiterhin im Verlauf der Fasern; in anderen Fällen war sie jedoch bis 2 , ja $3 \mu$ dick. Eine der Zwischenscheibe (beziglich einer halben Zwischenscheibe) entsprechende Schicht konnte ich am Faserende nie nachweisen, anch nicht in den letzterwähnten Fällen ${ }^{1}$ ). In diesen war auch von anderen Schichten (wie etwa einer zweiten Nebenscheibe) nichts zu entdecken.

An zweiter Stelle wurden Faserenden untersucht, welche im contrahirten Zustande fixirt worden waren. Man findet solche, namentlich auch unter den Abdominalmuskeln, sehr bäufig. Als allgemeines Resultat ergab sich, dass auch das letzte Muskelfach und speciell die terminale Schicht is otroper Substanz an der Verkürzung in der üblichen Weise Theil nimmt. Die bekannten charakteristischen Aenderungen - Abnahme des Volums, Zunahme des Lichtbrechungsvermögens, der Festigkeit, der Resistenz gegen Lösungsmittel, der Tinktionsfähigkeit, treten auch in der terminalen isotropen Schicht ein. Diese Aenderungen können, unter entsprechender Verkürzung, bis zum voll entwickelten Umkehrungsstadium führen. Häutig scheint bei stark contrahirten Fasern das Umkehrungsstadium gegen das Ende hin - etwa im Verlauf der letzten 3 oder 4 Fächer - in das Uebergangs- oder homogene Stadium auszulaufen. Doch zeigt nähere Untersuchung meist, dass dies eine Täuschung ist, verursacht durch die abnehmende Dicke der Faser und auch durch schiefe Uebereinanderlagerung der correspondirenden Fibrillenabschnitte, infolge schräger Lage der Endfläche in Bezug anf die Ebene des Gesichtsfeldes. Dass übrigens genug Fälle vorkommen, in denen das Faserende das wirkliche Uebergangsstadium zeigt und erst weiterhin das Bild der Umkehrung kommt, versteht sich von selbst. Ob in feineren Besonderheiten dem Faserende eigenthümliche Abweichungen vorkommen, wage ich bei der Schwierigkeit des Ge-

scheiben, beide gleich stark, doppelbrechend, Nebenscheiben und Zwischenscheiben, wie auch die kleine Sehne durchaus isotrop. Letztere ist, um nicht unnöthigen Raum wegzunehmen, auf etwa $2 / 3$ ihrer Länge abgekürzt. Das an die Muskelsubstanz sich anschliessende Drittel ist aher genau wiedergegeben, bis auf Zahl und Dicke der Sehnenfibrillen. Der Holzschneider hat diese etwa um das Doppelte zu zahlreich und zu schmal gezeichnet.

1) Dies ist beiläufig ein neuer Grund gegen die Annahme Merkels, dass die Zwischenscheibe aus zwei verkitteten "Endscheiben" bestehe. 
genstandes und der nicht sebr grossen Zahl hierauf genauer geprüfter Objecte nicht zu entscheiden.

In morphologischer Beziehung lehren die vorstehenden Thatsachen, dass als das eigentliche Element der quergestreiften Fibrille mit Recht der von Zwischenscheibe "zu Zwischenscheibe reichende Abschnitt betrachtet wird. Bisher war es immerhin noch möglich, wennschon nicht wahrscheinlich, dass die Mittelscheiben die Grenzen der natülichen elementaren Abschnitte bildeten, da auch in Bezug auf sie die Fibrille überall in ihrem Verlauf symmetrisch gebaut ist.

In physiologiseher Beziehung möge zuerst bemerkt sein, dass die nachgewiesene Art der Endigung eine solche ist, wie sie nach meiner Theorie der Contraction zu erwarten war. Da nach dieser die Verkürzung auf Quellung der anisotropen Theilchen (Inotagmen) durch Wasseraufnahme aus der isotropen Schicht beruht, musste auf die letzte vollständige Sehicht anisotroper Substanz noch eine halbe isotrope Schicht folgen, wenn die letzte Querscheibe noch in gehöriger Weise aktiv an der Verkürzung Theil nehmen sollte. Falls sich am Ende eine vollständige isotrope Schicht (Zwischenscheibe mit zwei Nebenscheiben und plasmatischen Lagen) gefunden hätte, so würde dies, als eine offenbare Materialverschwendung, ebenfalls mit meiner Theorie schwer zu reimen gewesen sein.

Die Abwesenheit einer, der Zwischenscheibe, bezüglich einer halben Zwischenscheibe, entsprechenden Lage am Faserende scheint zu beweisen, dass diese Schicht mit dem Contractionsvorgang in der angrenzenden Fachhälfte direct nichts zu schaffen hat. Ihr Fehlen entzieht zugleich den abenteuerlichen Vorstellungen einiger Autoren über eine ,attraktorische" Funktion derselben völlig den Boden. Vermuthlich liegt die physiologische Bedeutung der Zwischenscheibe wesentlich in ihrer Betheiligung am Erregungsvorgang, wofür u. a. schon ihre innigen anatomischen Beziehungen zu den Terminalfibrillen der motorischen Nervenfasern sprechen ${ }^{1}$ ). Aus diesem Gesichtspunkt ist ihr Fehlen am Faserende nur natürlich: denn hier giebt es nun nichts weiter zu erregen.

1) A. Foettinger, Sur les terminaisons des nerfs dans les muscles des insectes. Onderzoek, ged. in het physiol. labor. des Utrechtsche Hoogeschool. Derde Reeks. V, 1880, p. 293, PI. VII. 
536 Th. W. Engelmann: Ueber den Bau der quergestreiften Substanz etc.

Unsere anatomischen Ergebnisse scheinen endlich anch nicht ganz ohne Werth für das Verständniss der ausserordentlich grossen Empfindlichkeit der natïrlichen Faserenden in electromotorischer Bezi ehung gegen äussere Einwirkungen. Aus Gründen, die ich früher ${ }^{1}$ ) zusammengestellt habe, ist als sicher anzunehmen, dass die isotrope Substanz, neben der Rolle, die ihr - u. a. schon als Wasserbehälter für die anisotropen, contractilen Schichten für den Mechanismus der Verkürzung zukommt, wesentlich die eines Sitzes und Leiters des Erregungsvorganges spielt und insofern Quelle elektromotorischer Processe ist, in höberem Masse selbst als die anisotropen Schichten. Da sie nun, wie schon ihr ausserordentlich grosser, dem der Axencylinderfibrillen vergleichbarer Wassergehalt erwarten lässt, und mancherlei bekannte mikroskopischen Erscheinungen bestätigen, die am leichtesten veränderliche, im beweglichsten Gleichgewicht befindliche Schicht der Muskelsubstanz ist, muss ihre Lage an der Oberfläche der natïrlichen Faserquerschnitte im angegebenen Sinne förderlich wirken: schon verhältnissmässig geringe, den natürlichen Querschnitt treffende Eingriffe werden daselbst merkliche electromotorische Kräfte hervorrufen.

1) Dies Archiv, Bd. VII, p. 186. Bd. XI, p. 461 u. ff. - Compte rendu du Congrès period. internat. des scienc. médic. 1879. Amsterdam, van Rossem 1880 , p. 579 et suiv. 